\title{
Expression of $\alpha$-Klotho protein in human thyroid cancers an immunohistochemical study
}

\author{
Marek Pawlikowski', Hanna Pisarek ${ }^{2}$, Magdalena Borkowska ${ }^{3}$, Katarzyna Winczyk $^{2}$ \\ ${ }^{1}$ Department of Immunoendocrinology, Chair of Endocrinology, Medical University of Lodz, Poland \\ ${ }^{2}$ Department of Neuroendocrinology, Interdepartmental Chair of Laboratory and Molecular Diagnostics, Medical University of Lodz, \\ Poland \\ ${ }^{3}$ Department of General and Endocrinological Surgery, Copernicus Memorial Hospital, Lodz, Poland
}

\begin{abstract}
Introduction: The $\alpha$-Klotho protein was discovered as a gene controlling the process of aging, but further studies indicated that it also plays the role of a tumour suppressor. Although numerous studies were performed on the role of the $\alpha$-Klotho gene and protein in neoplasia, the data on $\alpha$-Klotho protein expression in thyroid cancers are very scarce. Our study presents the immunohistochemical investigation of $\alpha$-Klotho expression in benign and malignant thyroid tumours.

Material and methods: The material included samples of benign (nodular hyperplasia, follicular adenoma), differentiated (follicular and papillary) cancers and aggressive thyroid cancers of low differentiation grade. The samples were immunostained using two different monoclonal anti- $\alpha$-Klotho antibodies.

Results: From the two antibodies used in this study, one (EPR6856) reacted probably with the soluble form of Klotho and immunostained mostly the colloid filling thyroid follicles and intravascular or extravascular serum deposits. The other (A-9 antibody) immunostained the follicular epithelium in benign thyroid lesions as well as the epithelial tumoural cells in differentiated thyroid (follicular and papillary cancers). In the thyroid cancers of high malignancy, the immunostaining with A-9 anti- $\alpha$-Klotho antibody was (except in one case) negative or very weak.

Conclusion: Our results indicate that lowered expression of $\alpha$ - Klotho is involved in the process of thyroid neoplasia. (Endokrynol Pol
\end{abstract} 2019; 70 (3): 237-240)

Key words: $\alpha$-Klotho; thyroid malignancies; immunohistochemistry

\section{Introduction}

The $\alpha$-Klotho protein was discovered in mice in the 1990 s as a gene coding a protein exhibiting anti-aging properties. It was shown that its inactivating mutation leads to shortening of the lifespan and induces a multi-systemic syndrome resembling premature aging. The overexpression of this gene, in contrast, elongated lifespan [1]. The name Klotho refers to a Greek mythological goddess whose role was to cut the thread of human life. Further studies revealed the presence of Klotho in humans as well as its role as a tumour suppressor [2,3]. As the authors cited above indicate, the mechanism of anti-cancer activity of Klotho may depend on the inhibition of growth factors like IGF-1 and $\beta$-FGF. The anti-aging activity of Klotho may at least partially be similar since the bulk of evidence indicates a role of IGF-1 overexpression in aging [for review see: 4]. Other pathways in anti-cancer effects of Klotho were also taken into consideration [for review see: 5,6$]$. Although a wide range of human cancers were investigated for the expression of $\alpha$-Klotho, we found only one paper dealing with thyroid cancer [7]. In the above-mentioned paper it was shown that the overexpression of Klotho induces the inhibition of growth and increases apoptosis in follicular thyroid cancer cell lines in vitro. Moreover, in contrast to the numerous studies on $\alpha$-Klotho expression done by means of molecular biology techniques, immunohistochemical studies were very scarce, as well in cancers as in normal tissues [8, 9]. The present paper reports the immunohistochemical investigation of $\alpha$-Klotho in benign thyroid lesions and thyroid cancers.

\section{Material and methods}

\section{Tissue samples}

The project was approved by the Bioethical Committee of the Medical University of Lodz, number RNN/79/17/KE. The study materials comprised 53 (aged 23-89 years) archived thyroid tissues from 10 men (36-77; mean age: 59.6 years) and 43 women (23-89, mean age: 54.9 years). The investigated group consisted of 17 benign thyroid neoplasms (nodular goitres and follicular adenomas), 11 papillary cancers, 13 follicular cancers, and 12 malignant thyroid tumours (five poorly differentiated and seven anaplastic tumours). 


\section{Immunohistochemistry}

The tissue samples were fixed in $10 \%$ formalin and paraffin embedded. All procedures as well as blocking endogenous peroxidase and high-temperature antigen retrieval procedure with citrate buffer $\mathrm{pH} 6.0$ were performed. Immunohistochemical examination of $\alpha$-Klotho was performed using commercially available monoclonal antibodies (rabbit monoclonal antibody EPR6856, cat. no. ab181373 from Abcam and mouse monoclonal antibody A-9, cat. no. sc-515942 from Santa-Cruz). All the samples were immunostained with EPR6856 antibody. A-9 antibody was applied in 24 samples (three nodular hyperplasia, three follicular adenomas, seven follicular cancers, two papillary cancers, and nine anaplastic or poorly differentiated thyroid cancers). The primary antibodies were applied at dilutions 1:250 (EPR6865) and 1:100 (A-9) with $4^{\circ} \mathrm{C}$ overnight incubation. After washing steps, the sections were treated with DAKO Real EnVision + Detection System, Peroxidase/DAB+ Rabbit/Mouse (cat. no. K5007, DAKO). The immunoreaction was visualised with $3.3^{\prime}$-diaminobenzidine (DAB) solution.

\section{Results}

\section{Immunostaining with EPR6856 antibody}

In the benign thyroid neoplasms positive immunostaining was observed in the follicular colloid in 7/9 cases (Fig. 1) but not in the follicular epithelium. In one case a positive immunostaining was noticed in the intravascular serum deposit, and in other case a weak immunoreaction was also found in the stromal cells. In the follicular thyroid cancer group there was no positive immunostaining in either cancer tissues or in blood vessels, but positive immunoreactivity in the colloid of single follicles was observed. In one case of follicular adenoma, positive immunoreactivity in some inflammatory infiltration cells and in exudate fluid was observed. In the papillary cancers a negative immunoreaction in $7 / 11$ cancer tissues with positive reaction in the colloid of single follicles at the periphery of the tumour was found. Therefore, in the two cases several immunopositive cells within the tumoural tissue were observed (Fig. 2). The

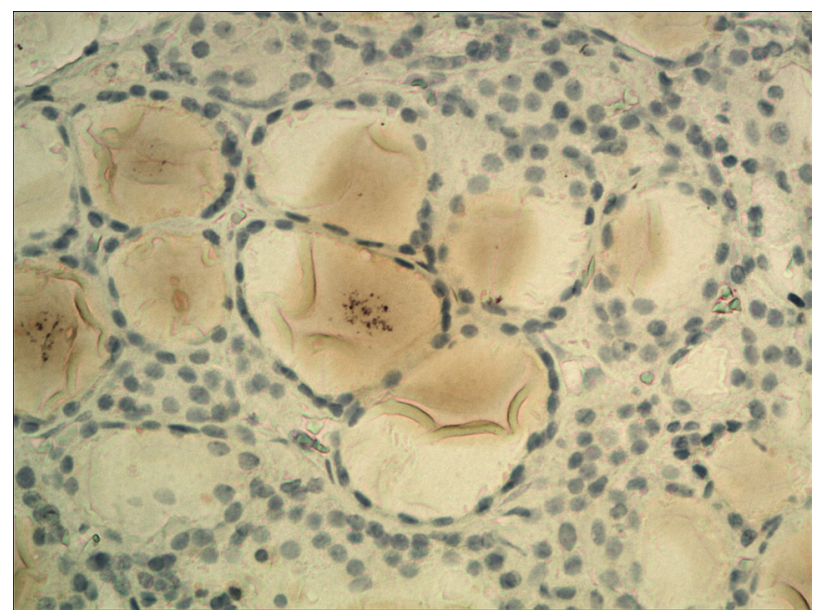

Figure 1. Nodular thyroid hyperplasia. Negative immunoreaction with anti- $\alpha$-Klotho antibody EPR6856 in follicular cells and positive in the colloid

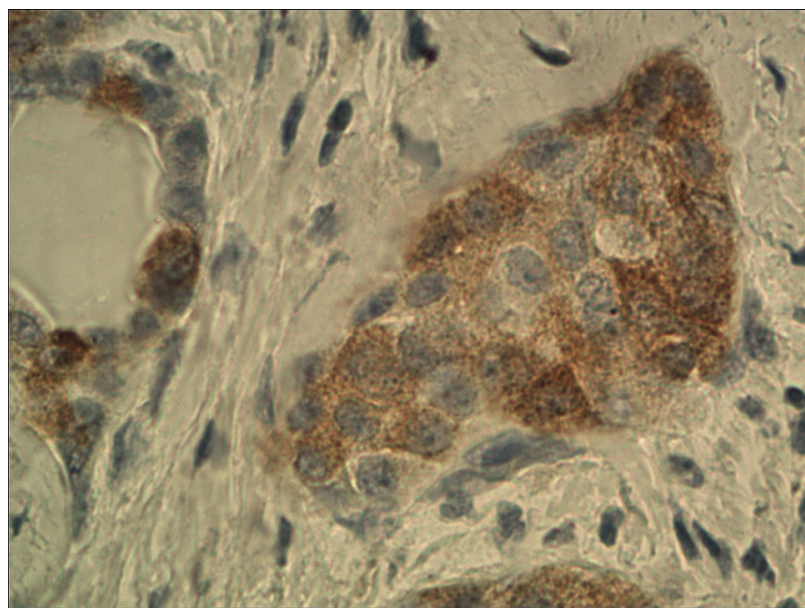

Figure 2. Papillary thyroid cancer. Positive $\alpha$-Klotho immunostaining in tumoural cells (EPR6856 antibody)

staining in the blood vessels was negative. No positive immunostaining was found in the aggressive thyroid cancer tissues (PDTC and ATC). Two of the PDTC samples exhibited $\alpha$-Klotho immunopositivity inside the intravascular serum deposit but not vessel walls and in the inflammatory infiltration. Weak immunoreaction was observed in the single stromal cells of one PDTC sample.

\section{Immunostaining with A-9 antibody}

In the benign lesions (nodular hyperplasia or follicular adenoma) in all the samples a strong positive immunostaining in the vast majority of follicular epithelia was observed (Fig. 3). In contrast to EPR antibody, the immunostaining of colloid is very rare (present in a few follicles in only one case of nodular hyperplasia). No immunoreaction was observed in blood vessel content. In differentiated thyroid cancers (papillary and follicular cancers) we found strong immunostaining of tumoural cells in all but one sample (Fig. 4 and 5). In one case (follicular cancer) the immunoreaction was negative except a few strongly positive follicles. In poorly differentiated or anaplastic cancers the immunoreaction with anti- $\alpha$-Klotho A-9 antibody was negative or very weak (Fig. 6), except in one case in which it was moderate.

\section{Discussion}

In our study we used two different anti- $\alpha$-Klotho monoclonal antibodies, with clearly distinct results. In the case of EPR6856 antibody the immunoreaction concerns mostly the colloid filling the thyroid follicles. The immunoreaction is also shown in serum deposits inside blood vessels. Such a localisation is compatible with the customer's opinion (based on the western blot study) that the antibody EPR6856 reacts with a soluble $\alpha$-Klotho but not with the full length $\alpha$-Klotho molecule 


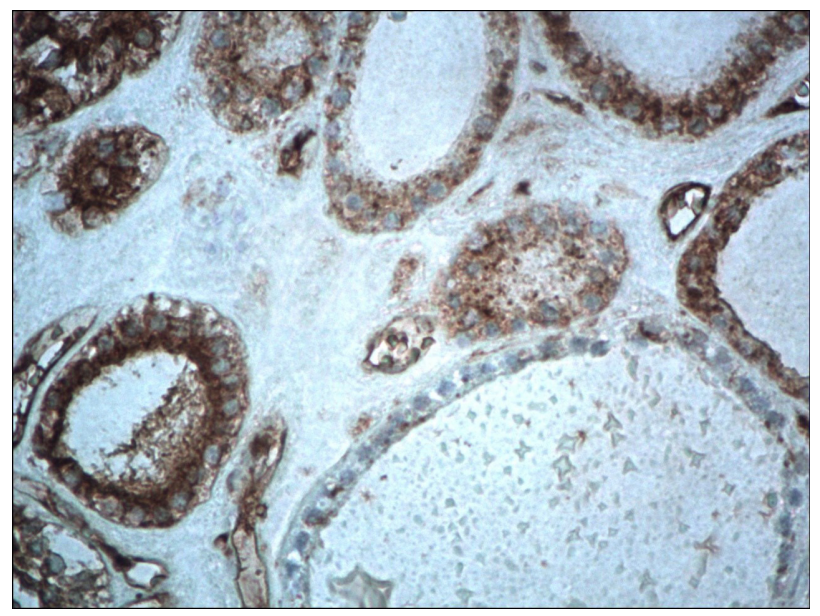

Figure 3. Thyroid nodular hyperplasia. Strong immunostaining with anti- $\alpha$-Klotho A-9 antibody in follicular epithelial cells

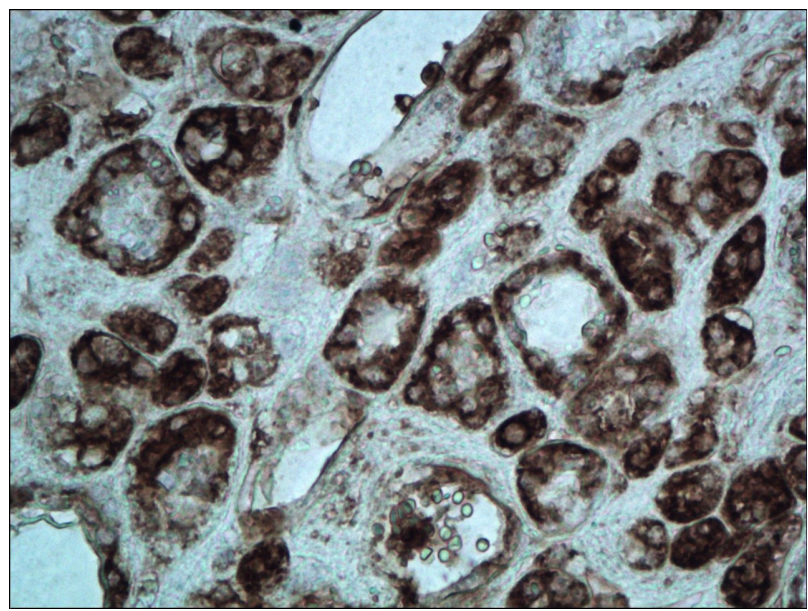

Figure 4. Follicular thyroid cancer. Strong immunostaining with anti- $\alpha$-Klotho A-9 antibody in follicular epithelial cells

[10]. The explanation of positive immunoreaction in cytoplasm of certain tumoural cells is not so simple. However, we have to remember that soluble $\alpha$-Klotho sequence is included as a KL-1 fragment in the whole $\alpha$-Klotho molecule and is expressed as the result of alternative splicing but can be released also by proteolytic action from the full-length protein [11, 12]. When the A-9 antibody was used, the intensive immunostaining in benign thyroid lesions concerns the follicular epithelium. It is in agreement with the observations of normal human thyroid by Lim et al. [9]. In thyroid cancers of low malignancy, i.e. papillary and follicular cancers, immunostaining with A-9 antibody occurs in almost all tumoural cells. In cases of highly malignant, poorly differentiated, and anaplastic thyroid cancers (except one sample) the immunoreaction with A-9 anti- $\alpha$-Klotho antibody was negative or very weak. This is compatible with many data showing the down-regulation of the

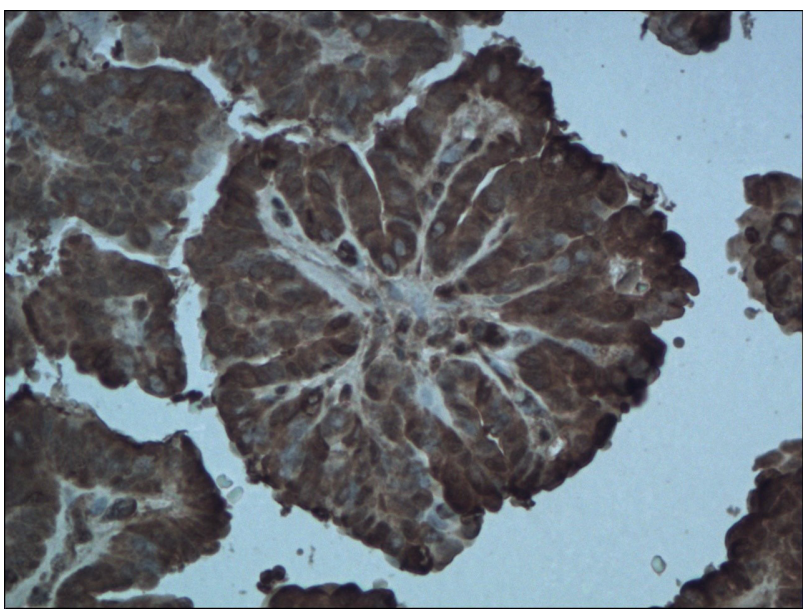

Figure 5. Papillary thyroid cancer. Strong immunostaining with anti- $\alpha$-Klotho A-9 antibody in tumoural cells

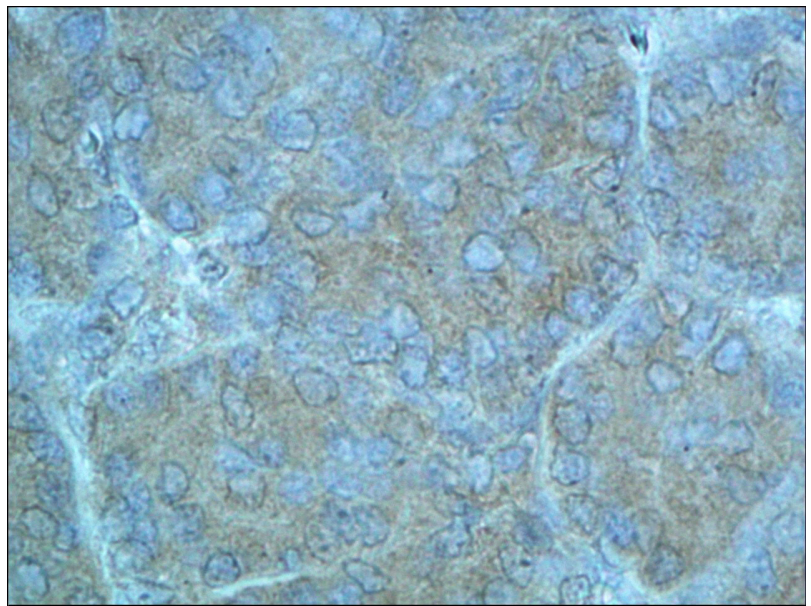

Figure 6. Poorly differentiated thyroid cancer. Very weak immunostaining with anti- $\alpha-K l o t h o ~ A-9$ antibody in tumoural cells

$\alpha$-Klotho expression in such human neoplasms as pancreatic [2,13], breast [3], oral squamous cell [8], cervical [14], and gastric cancer [15]. Recently, it was also found that a protein similar to $\alpha$-Klotho, namely $\beta$-Klotho, is decreased in the blood serum of patients with thyroid cancers vs. patients with benign nodular goitre [16].

The immunohistochemical examination of $\alpha$-Klotho protein expression in thyroid samples could offer the possibility to differentiate between low- and high-malignancy tumours. On the other hand, the immunostaining of Klotho protein does not allow the differentiation between benign and low-malignancy cancerous lesions (e.g. between follicular adenomas and follicular cancers). These suggestions need further studies on larger material. To conclude, our study shows that decreased expression of the $\alpha$-Klotho protein is involved in thyroid cancerogenesis in case of highly malignant cancers with low grade of differentiation. 


\section{Funding}

The study was supported by Medical University of Lodz — grant No. 503/5-020-02/503-51-001.

\section{References}

1. Kuro-o M, Matsumura Y, Aizawa H, et al. Mutation of the mouse klotho gene leads to a syndrome resembling ageing. Nature. 1997; 390(6655): 45-51, doi: 10.1038/36285, indexed in Pubmed: 9363890.

2. Abramovitz L, Rubinek T, Ligumsky H, et al. KL1 internal repeat mediates klotho tumor suppressor activities and inhibits bFGF and IGF-I signaling in pancreatic cancer. Clin Cancer Res. 2011; 17(13): 4254-4266, doi: 10.1158/1078-0432.CCR-10-2749, indexed in Pubmed: 21571866.

3. Wolf I, Levanon-Cohen S, Bose S, et al. Klotho: a tumor suppressor and a modulator of the IGF-1 and FGF pathways in human breast cancer. Oncogene. 2008; 27(56): 7094-7105, doi: 10.1038/onc.2008.292, indexed in Pubmed: 18762812

4. Bartke A, Kopchick J, Dominici F, et al. IGF-1 and Insulin Signaling in the Control of Longevity. In: Endocrine aspects of successful aging: genes, hormones and lifestyles. P. Chanson, J. Epelbaum, S. Lamberts, Y. Christen (ed) . Springer Berlin 2004: 19-33.

5. Zhou X, Wang X. Klotho: a novel biomarker for cancer. J Cancer Res Clin Oncol. 2015; 141(6): 961-969, doi: 10.1007/s00432-014-1788-y, indexed in Pubmed: 25086986.

6. Rubinek T, Wolf I. The role of alpha-klotho as a universal tumor suppressor. Vitam Horm. 2016; 101: 197-214, doi: 10.1016/bs.vh.2016.03.001, indexed in Pubmed: 27125743.

7. Dai D, Wang Qi, Li X, et al. Klotho inhibits human follicular thyroid cancer cell growth and promotes apoptosis through regulation of the expression of stanniocalcin-1. Oncol Rep. 2016; 35(1): 552-558, doi: 10.3892/or.2015.4358, indexed in Pubmed: 26531219.

8. Adhikari BR, Uehara $\mathrm{O}$, Matsuoka $\mathrm{H}$, et al. Immunohistochemical evaluation of Klotho and DNA methyltransferase 3a in oral squamous cell carcinomas. Med Mol Morphol. 2017; 50(3): 155-160, doi: 10.1007/s00795-017-0156-9, indexed in Pubmed: 28303350.

9. Lim K, Groen A, Molostvov G, et al. $\alpha$-klotho expression in human tissues. J Clin Endocrinol Metab. 2015; 100(10): E1308-E1318, doi: 10.1210/jc.2015-1800, indexed in Pubmed: 26280509.

10. Abcam. Product datasheet. Anti-Klotho antibody [EPR 6856] ab181373.

11. Matsumura Y, Aizawa H, Shiraki-Iida T, et al. Identification of the human klotho gene and its two transcripts encoding membrane and secreted klotho protein. Biochem Biophys Res Commun. 1998; 242(3): 626-630, indexed in Pubmed: 9464267.

12. Szymczyk A, Forma E. Structure and function of Klotho protein [in Polish]. Folia Medica Lodzensia. 2012; 19: 151-187.

13. Jiang B, Gu Y, Chen Y. Identification of novel predictive markers for the prognosis of pancreatic ductal adenocarcinoma. Cancer Invest. 2014; 32(6): 218-225, doi: 10.3109/07357907.2014.905586, indexed in Pubmed: 24745611

14. Lee J, Jeong DJ, Kim J, et al. The anti-aging gene KLOTHO is a novel target for epigenetic silencing in human cervical carcinoma. Mol Cancer. 2010; 9: 109, doi: 10.1186/1476-4598-9-109, indexed in Pubmed: 20482749

15. Wang L, Wang $X$, Wang $X$, et al. Klotho is silenced through promoter hypermethylation in gastric cancer. Am J Cancer Res. 2011; 1(1): 111-119, indexed in Pubmed: 21969138.

16. Motylewska E, Stępień T, Borkowska M, et al. Alteration in the serum concentrations of FGF19, FGFR4 and $\beta$ Klotho in patients with thyroid cancer. Cytokine. 2018; 105: 32-36, doi: 10.1016/j.cyto.2018.02.013, indexed in Pubmed: 29438906. 